\title{
ULTRA-HIGH EFFICIENCY LIGHT-EMITTING-DIODE ARRAYS
}

\author{
I. Schnitzer, E. Yablonovitch, A. Ersen, A. Scherer, $\nmid$ C. Caneau, $\nmid$ and T. J. Gmitter \\ UCLA, Department of Electrical Engineering 405 Hilgard Ave., Los Angeles, CA 90024-159410 \\ VOICE (310)206-1034, FAX (310)206-8495,E-MAIL schnit@ee.ucla.edu \\ $\dagger$ Bellcore, 331 Newman Springs Rd., Red Bank, NJ 07701-7040
}

High efficiency light-emitting-diodes (LED's) are desired for many applications such as displays, printers, shorthaul communication, and opto-electronic computer interconnects. However, there is an enormous gap between the theoretical efficiency of LED's and their actual efficiency. The internal quantum yield, $\eta$, of good quality double heterostructures can exceed $99 \%$, as we have demonstrated recently. ${ }^{1}$ On the other hand run-of-the-mill commercial LED's are usually only a few percent efficient. The reason for this long-standing shortfall is the difficulty for light to escape from high refractive index semiconductor. A mere $2 \%$ of the internally generated light is coupled into free space through the $16^{\circ}$ escape cone, the rest suffering total internal reflection and risking re-absorption. The present commercial state-of-the-art, $\sim 20 \%$ external efficiency in AlGaAs-based LED's, is achieved by growing a thick transparent semiconductor superstrate, and total substrate removal in a particularly clean, low-loss, optical design which can add greatly to the cost.

The key to increasing the escape probability is to give the photons multiple opportunities to find the escape cone. This requires angular randomization or scrambling of the light rays. One way to do this is by photon high selfabsorption, and a very clean, non-dissipative optical design. In ref. 1 we used the epitaxial liftoff (ELO) technique ${ }^{2}$ to mount thin film heterostructures on high reflectivity surfaces. It was found that while the photon re-cycling can yield an ultrahigh external quantum efficiency ( $72 \%$ ), it is very susceptible to parasitic loss mechanisms (free carrier absorption in doped layers, for example) or slight degradation in the internal quantum efficiency. A more practical approach, and the one which we propose here is the angular randomization by elastic scattering of the photons from a textured semiconductor surface.

Our approach has two components: i) Separation of thin-film heterojunctions from the growth substrate using the ELO technique, and ii) Nano-texturing of the thin-film semiconductor interface by natural lithography. ${ }^{3}$ The implementation of these two components leads to a thin film $(<1 \mu \mathrm{m})$ geometry in which one side of the heterostructure is facing a high quality reflector while the other surface, the semiconductor/air interface, is textured to act as a strong scatterer. This geometry employs the statistical mechanical concept of phase-space filling by angular randomization. We invoke a statistical approach ${ }^{4}$ to model the smooth and textured film goemetries. It is concluded that the textured film geometry can boost the external efficiency to $50 \%$ or more, while relieving the demand for the utmost material quality, high self-absorption, and low parasitic losses, as required by the smooth film geometry of Ref. 1 .

The actual LED structure is a conventional $\mathbf{n}^{+}$-AlGaAs $/ \mathrm{p}-\mathrm{GaAs} / \mathrm{p}^{+}$-AlGaAs double heterostructure, grown over a $0.05 \mu \mathrm{m}$ thick AlAs release layer by organo-metallic chemical vapor deposition. Large LED's arrays are fully processed from these wafers, then separated from the GaAs substrate by epitaxial liftoff, and Van der Waals bonded by surface tension forces onto large area dielectric coated Au mirrors. To minimize the likelihood that light will be parasitically absorbed at the Ohmic contacts, a significant problem in all LED's, we have employed the concept of current crowding. Carriers are injected into a central region between the two Ohmic contacts, but reasonably distant from either contact. The final processing step is the texturing of the light emitting region. The surface of the LED is coated by a randomly close-packed array of polystyrene spheres, $0.2 \mu \mathrm{m}$ diameter, using the surface forces between the charged spheres and the semiconductor. The spheres then act as an etch mask for a $\mathrm{Cl}_{2}$ assisted $\mathrm{Xe}^{+}$ion beam etching, about $0.17 \mu \mathrm{m}$ deep.

The light versus current characteristics of these LED's have been measured and modeled. We have observed 9\% external quantum efficiency from untextured LED's array transforming into $30 \%$ external quantum efficiency following the surface texturing treatment. We conclude that by employing the principle of phase-space-filling in an improved device geometry, 56\% efficient LED's arrays can be expected. Such LED's are simpler and more reliable than lasers. Unlike lasers they are thresholdless, yet they offer comparable external efficiency. Moreover, the principles and the device geometry that we are proposing can be applied to other semiconductor material systems to obtain very bright visible thin film, top processed, LED's arrays as well. It is clear that the availability of $30 \%-50 \%$ efficient, low-cost, reliable, visible LED's would change dramatically the appearance of our homes, offices, and streets.

1. I. Schnitzer, E. Yablonovitch, C. Caneau, and T. J. Gmitter, Appl. Phys. Lett. 62, 131 (1993).

2. E. Yablonovitch, T. J. Gmitter, J. P. Harbison, and R. Bhat, Appl. Phys. Lett. 51, 2222 (1987).

3. H. W. Deckman and J. H. Dunsmuir, Appl. Phys. Lett. 41, 377 (1982).

4. E. Yablonovitch, J. Opt. Soc. Am. 72, 899 (1982). 
Figure 1: Typical photon trajectory in the thin epi-liftoff heterostructure: $a$. In the smooth structure the photons trajectories are randomized by the selfabsorption/re-emission events, represented by dots. $b$. In the textured film, angular randomization is achieved by strong surface scattering.

Figure 2: Schematic cross section of an epi-liftoff, textured LED, Van der Waals bonded onto an $\mathrm{SiO}_{2}$ coated metallic reflector. The spatial distribution of injection current is represented by the curve on the graph $j(x)$ versus $x$. Current crowding is obtained due to the different conductivity of the $\mathrm{n}^{+}$and $\mathrm{p}^{+}$contact layers, in order to minimize absorption losses at the metallic electrodes. The width of the textured area is about 50 times the film thickness.

Figure 3: Natural Lithography Polystyrene spheres, $0.2 \mu \mathrm{m}$ diameter, coat the surface of the LED in a randomly close-packed array. The spheres then act as an etch mask for a $\mathrm{Cl}_{2}$ assisted $\mathrm{Xe}^{+}$ion beam etching, about $0.17 \mu \mathrm{m}$ deep. The spheres can be easily washed away to leave a clean high refractive index textured surface which enhances the outcoupling of light.

Figure 4: $\log$-Log plot of the measured total light output versus injection current, for typical textured and untextured (smooth) LED's, as indicated. The linear fits have slopes 1 , and their intercepts suggest the indicated external quantum efficiency.
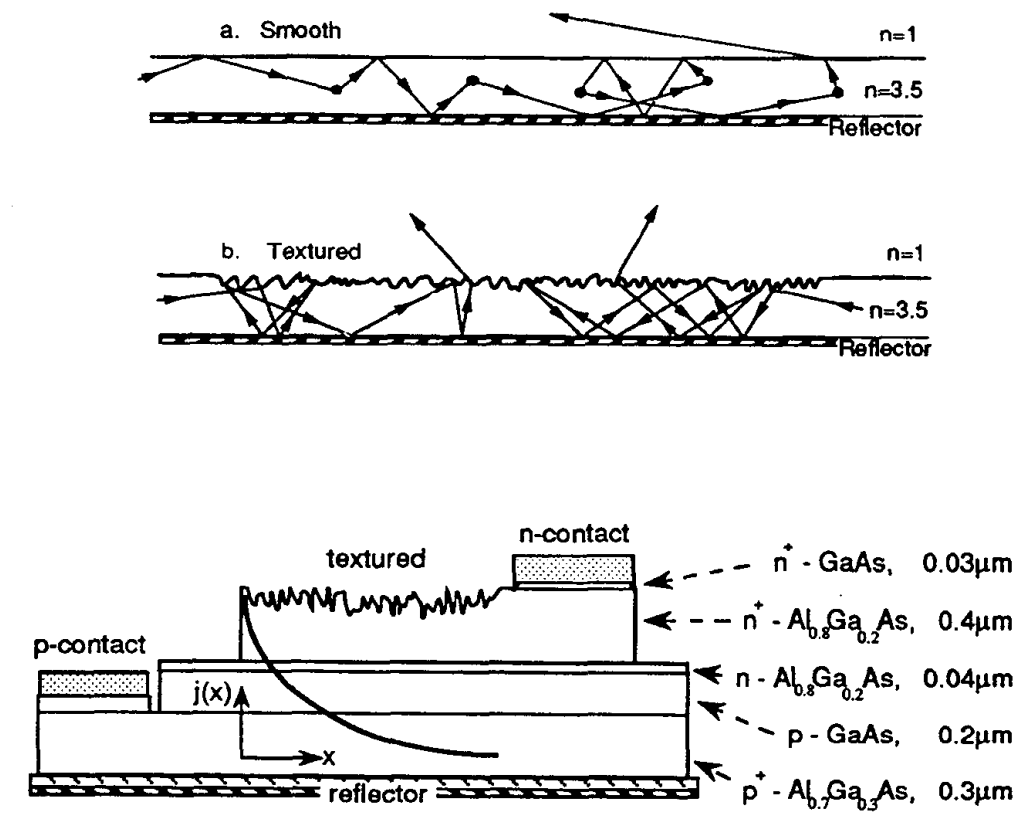

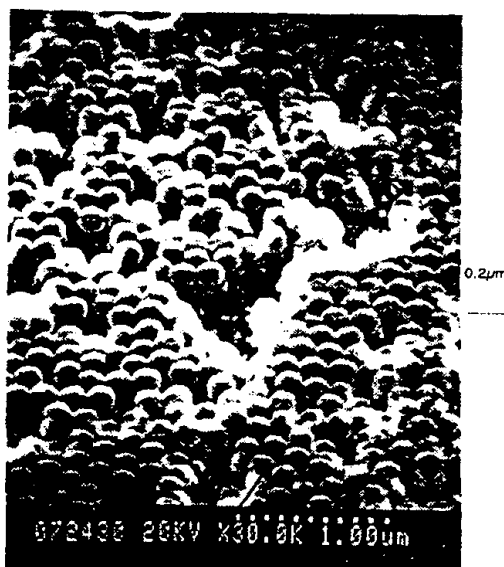

Poly-spheres

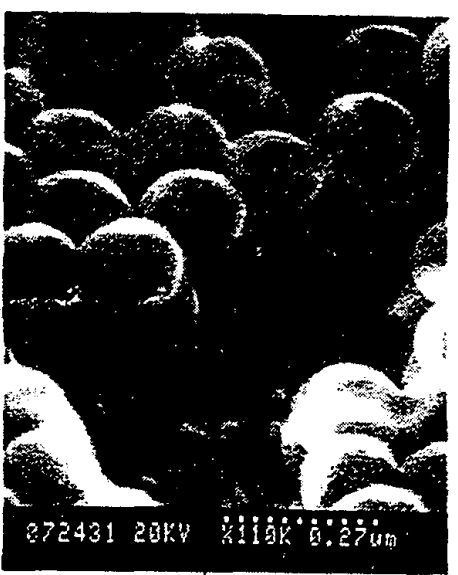

Semiconductor

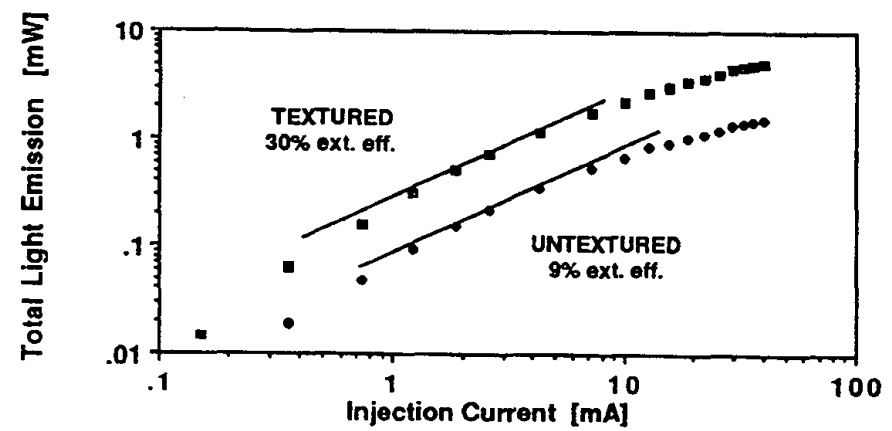

